\title{
AUTOMATED TRACKING OF STRUCTURAL STEEL MEMBERS AT THE CONSTRUCTION SITE ${ }^{1}$
}

\author{
Karen M. Furlani and Lawrence E. Pfeffer \\ Construction Metrology and Automation Group \\ National Institute of Standards and Technology \\ Gaithersburg, MD 20899, USA \\ kfurlani@nist.gov.pfeffer@nist.gov
}

\begin{abstract}
This paper presents further development and experimental testing of CompTRAK, a prototype system for identification and spatial tracking of structural steel components on a live construction site. Comp-TRAK is a web-based system for rapid tracking of construction components with compact, field-rugged sensors and computers, interoperability protocols for data transmission, and a 3-D site visualizer that reflect the current state of tracked components on the construction site. The result is a graphicallydriven system that provides real-time identification, position, and orientation data about components to users in the field and at remote locations.
\end{abstract}

Keywords: bar code, component tracking, construction, laser positioning system, metrology, real-time tracking, RFID, wireless data transfer.

\section{INTRODUCTION}

The Construction Metrology and Automation Group (CMAG) at the National Institute of Standards and Technology (NIST) has on-going research in real-time construction component tracking to address the problem of identifying and tracking discrete construction components and sub-assemblies on a construction site. Discrete components, in the context of the present discussion, are manufactured prefabricated singular items, such as structural steel, which carry a means of identification, such as a bar code label.

Although many technologies exist to aid in the automation of component tracking systems, their uses are limited by a lack of construction industry standards supporting inter-operability between various hardware and software systems. Additionally, many products are not designed to function and survive in the hostile and dynamic environment of a industry overcome these obstacles and achieve a fully integrated and automated tracking system, the CMAG is developing and testing Comp-TRAK, a prototype system for tracking manufactured components at the jobsite. The Comp-TRAK system involves the use of RFID and bar code identification systems, 3D longrange coordinate measurement technologies, local data processing, wireless communication, networking, temporal project databases, web-based typical construction site. To help the construction

data analysis, and 3D user interfaces to provide realtime access to part status updates at the jobsite and to remote, off-site office users. With this approach, CMAG is focusing on developing the technical infrastructure to support real-time tracking of subassemblies and components at the jobsite.

\section{PROJECT SCOPE}

In earlier work, we proposed an overall system architecture [1] and developed a prototype system that experimentally demonstrated 3D spatial tracking of discrete components in real-time and under controlled conditions to viewers at a remote location over a km away [2]. Most recently, the prototype system was field tested at the Building 205 Emission Control System (ECS) addition - a US\$6 million project on the NIST, Gaithersburg campus. A subset of the ECS structural steel framework was tracked. This paper discusses integration and implementation issues encountered while developing and testing the Comp-TRAK system for data collection on a live construction site.

\section{COMP-TRAK SYSTEM}

\subsection{System Overview}

\footnotetext{
${ }^{1}$ Official constribution of the National Institute of Standards and Technology; not subject to copyright in the United States
} 
Structural steel components scheduled for arrival on the construction site are tagged with bar code and/or radio-frequency identification (RFID) tags at the galvanizing plant prior to shipment. Via a webbased graphical user interface, the identification information encoded on the tag is scanned directly into a ruggedized, wearable computer and used to query a project database for additional information relating to the scanned item, including a $3 \mathrm{D}$ CAD model of the part. This model, coupled with userfriendly web browsing software, guides field workers through the measurement of key fiducial points using a long-range $3 \mathrm{D}$ coordinate measurement system to obtain $\mathrm{x}, \mathrm{y}, \mathrm{z}$ point data. We use the term fiducial point to mean pre-specified points, typically at readily identified features of interest such as corners. The fiducial points selected comprise a sufficient set of locations to establish the position and orientation of a component in 3-D space. The collected part ID and $\mathrm{x}, \mathrm{y}, \mathrm{z}$ fiducial point data is sent wirelessly to a remote server hosting a part locator service, which determines the object's position and orientation in the job site's global coordinate system (see figure 1.) The database then uses this data to track the current state of the object.

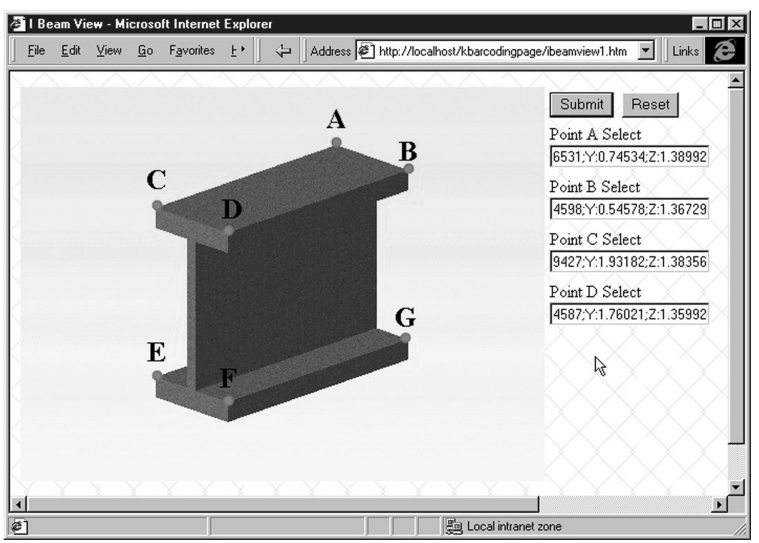

Figure 1: After scanning a part's bar code or RFID tag for the unique ID, the project database returns a 3D model with visual queues for the location of key fidicial points. Above, the field inspector has measured coordinate data for Points A-D, which are then wirelessly relayed to the part locator service.

\subsection{Field Data Collector}

The field data collector (see figure 2), initially developed and tested in a controlled laboratory environment, was modified and upgraded for the field testing at the Bldg. 205 ECS addition. Originally hosted on an IBM laptop computer, the data collection system was re-configured for use with a VIA II belt-wearable computer [3]. A PCMCIA RangeLAN2 Ethernet Adapter by Proxim and a Quatech DSP100 Dual Serial I/O Card provide wireless communications and two additional RS232D serial ports, respectively.

For part identification, a bar code laser scanner with built-in keyboard wedge software integrates via the USB port, and a RFID integrates via a RS-232D serial port. To measure 3D coordinate data for fiducial point collection, a long range (50 $\mathrm{m}$ radius) Vulcan 3D measurement system from ArcSecond, Inc. [4] integrates via a RS232 serial port using a C program written at NIST to interface the field computer with the Vulcan sensing tool. The Vulcan system creates a 3-D measuring environment within the intersecting area of two rotating fanned laser beam transmitters. A mobile "receiving" measurement tool collects coordinate point data within the working area created by the transmitters.

The entire web-based user interface runs through an Internet Explorer 4.1 graphical user interface written in Microsoft FrontPage98, driven by VisualBasic Scripts. Active Server Pages interact with the project database, and a LiveView [5] Common Gateway Interfaces (CGI) script provides the protocol for the transmission of both the part ID and its coordinates to the part locator service for position and orientation determination.

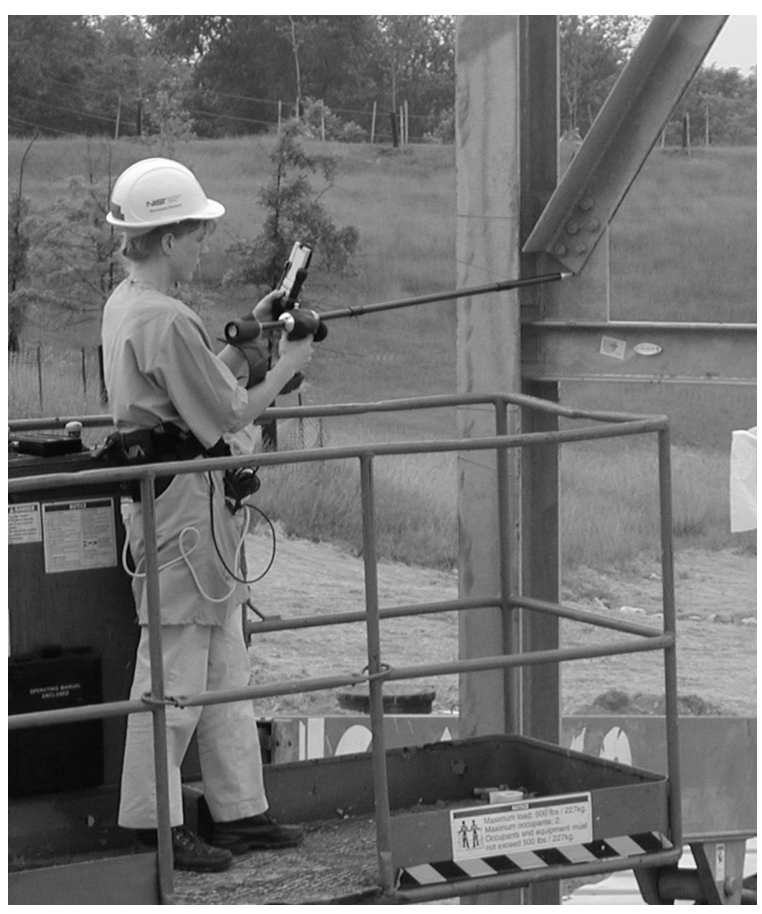

Figure 2. Field operator collecting fiducial point coordinate data with the Vulcan receiving tool integrated with the VIA belt-wearable computer and bar code scanner. A manlift was used to facilitate data collection once the steel tower was erected and installed is its final location.

\section{FIELD TEST}

\subsection{Part Identification}


A subset of the ECS structural steel framework, Tower \#2 (see figure 3), was tagged with bar codes at the galvanizing plant. Each bar code tag has the unique part identifier encoded in Code 3-of-9 symbology and also printed in human readable font along with some additional descriptive information related to the tagged part.

During pre-construction meetings with the steel fabricator, it was verified that piece marks, typically stamped on a steel member at the fabricators for identification purposes, would be located on the far lside of a steel part as typically shown on the fabricator's drawings. Since this provided an easily findable and repeatably consistent location on the steel, the bar code tags were attached adjacent to each steel part's piece mark to aid yard workers attaching the bar codes at the fabricator as well as field workers trying to scan the tags at the construction site

A member of the CMAG research team visited the yard crews at the galvanizing plant to explain the purpose and desired placement of the bar code tags. After the first couple tags were attached, the remainder were left with crew leader to finish. A field inspection of the tagged steel after deliver to the jobsite yielded no instances of unmarked or mismarked steel. During a follow-up conversation, the crew leader remarked that the tagging process was very simple and took little time to accomplish, noting that it only involved matching up the tags (which were organized in numerical order) with the piece marks on the steel.

The bar code tags, printed on "Stick Tuff" by Integrated Labeling Systems, Inc., survived the full length of the project; they stayed attached and readable from the time the steel parts were tagged at the galvanizers through final erection at the job site. As mentioned above, each steel part was tagged with a bar code tag located adjacent to the fabricator's stamped piece mark. Although this simplified where to attach and look to scan a tag, there were numerous times when access to the one tag was difficult, if not altogether impossible, due to the stacking of steel in the laydown area, and occasionally, the orientation of the tag once a part was installed. In future applications, attaching more than one tag on a steel part will provide a field operator with additional options in case of inaccessible or damaged tags.

Attempts to use RFID tags for part identification were not successful due to difficulties in permanently attaching the tags to the structural steel members. Encased in a plastic housing, the two options for attachment are via wire or epoxy. Due to the nature of most steel members, there is no good way to attach RFID tags using wire. Although bolt holes might work initially, they would become ineffective at the time of erection. Attaching the tags with epoxy resulted in all the tags falling off or being easily knocked off within days of their attachment.

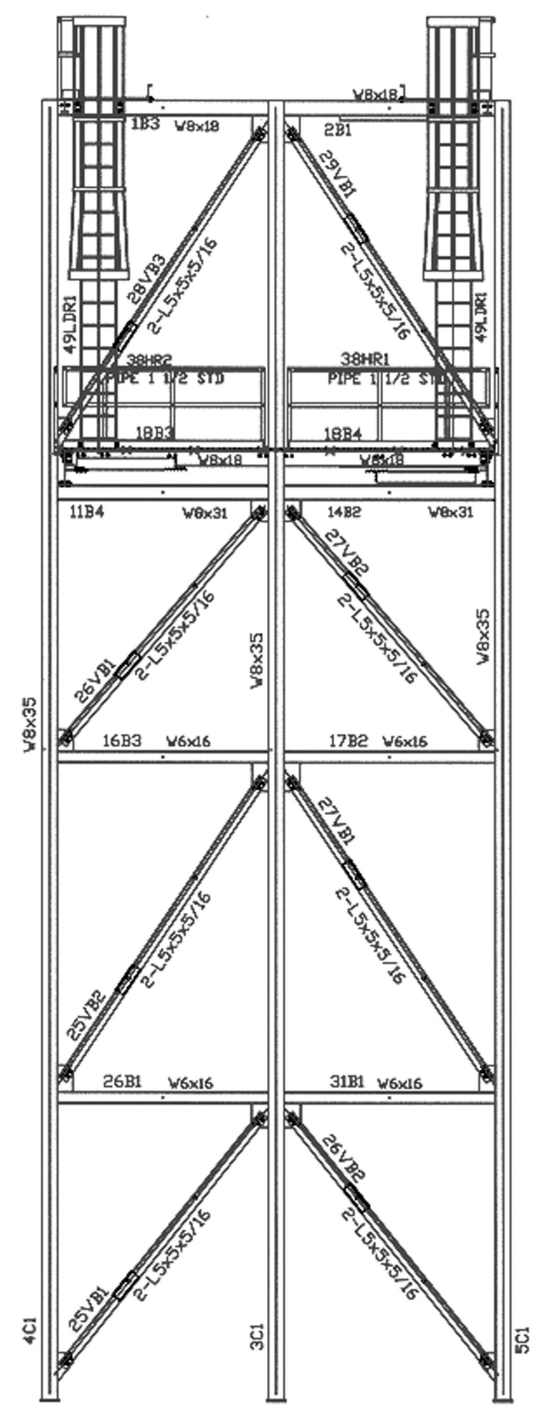

Figure 3. Elevation view of Bldg. 205 ECS Tower $\# 2$. The columns, angles and beams were tracked during the field test. The fabricator's ID shown adjacent to each steel member served as the basis for the unique part ID assigned to each part in the project database. The unique ID was encoded on the bar code and RFID tags for auto identification at the job site and used to query the project database to return the VRML model of the part to guide users through fiducial point measurements.

\subsection{Fiducial Point Assignment Convention}

Two basic structural steel shapes were tracked in the Tower \#2 subset: W shapes (I-beams and columns) and angles (see figure 4.) A standard set of default fiducial point locations were established for each of the three basic geometric shapes. The upper, left-hand corner above the piece mark was designated fiducial point \#1 on all the parts. Since the piece mark was always stamped on the steel in the same location, this provided a relatively simple methodology for the field test without requiring that a 
separate VRML model be created for each individual steel part. In general, this methodology worked well with the sampling of tracked steel, since the only geometric difference between structural steel pieces with identical shapes is length. However, difficulties arose when steel parts arrived with pre-attached plates or were erected into groups on the jobsite, and when access to at least three of the pre-defined fiducial points was not possible. Work in follow-on years will seek to identify potential solutions to such complex measurement issues as when certain parts are identical and interchangeable with others on a project, when parts are symmetrical, and when parts are theoretically identical with others according to fabricators drawings, but have been shortened due to a field clearance (see Section 5.0.)

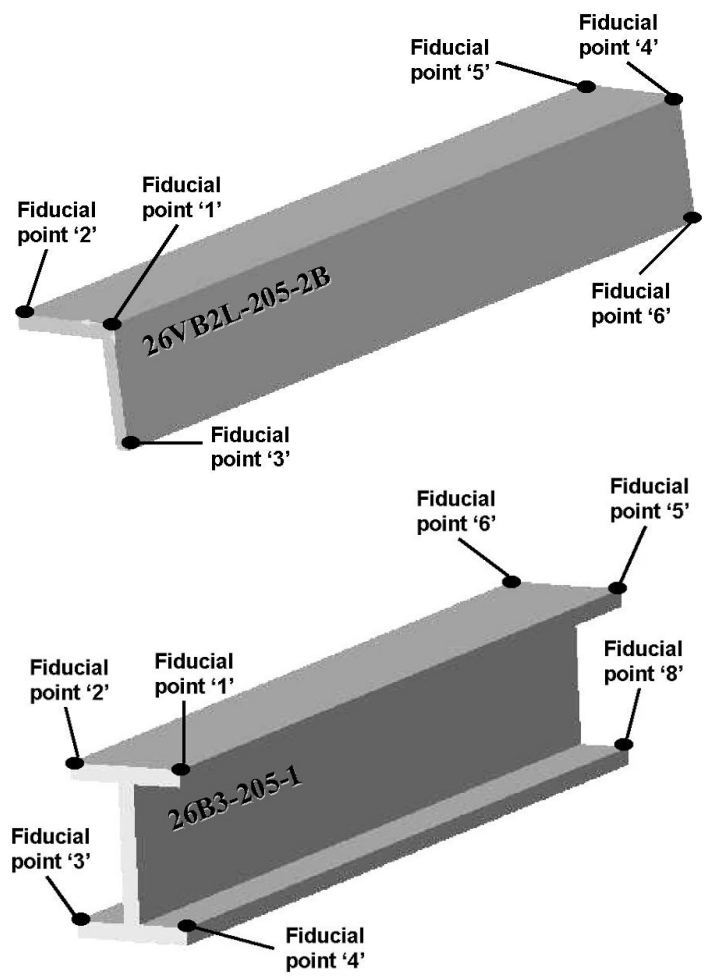

Figure 4. An enhanced VRML model of the structural steel angle (top) and I-Beam (bottom) with fiducial point markings and fabricator's steel mark help guide field workers through the acquisition of $\mathrm{x}, \mathrm{y}, \mathrm{z}$ coordinate data for a minimum of three fiducial points for position and orientation determination of a part at the jobsite.

\subsection{Coordinate Measurement System Registration}

To obtain registered 3-D coordinate measurement data using the Vulcan system, a known coordinate frame must first be established. There are two basic options available, either:
1) The field measurement sensor can be configured to transform its measurements into a known, sitereference frame using the Vulcan re-section calibration; or,

2) Data sufficient to uniquely determine the sensor's working coordinate frame can be recorded, so that data in the working frame can be transformed after the field measurements are taken using the Vulcan's quick calibration.

The re-section calibration method requires the collection of four points known in the desired frame and taken within the area where field measurements will be taken. These points must be within range and line-of-sight of the transmitters and accessible by the field inspector using the field measurement tool at all times during the project. In addition, the field measurement tool must be levelled when collecting these points. While this method allows the transmitters to be set-up at unknown locations on an as needed basis, it would be nearly impossible to guarantee continued access to a set of known survey points within the construction area without continually surveying new points as construction progresses through the lifecycle of the project. In addition, the likelihood that equipment and materials would block access and/or line-of-sight during measurements is fairly high.

Due to these limitations, the quick calibration method was used during the field test. This method establishes a frame based on the location and orientation of the transmitters. While this still requires a known set of locations for the transmitters, and requires that the transmitter serving as the origin be levelled, they can be located outside the actual work area and remain undisturbed during construction.

To obtain known points for the transmitter locations, ten benchmarks were established to provide adequate coverage for measurements in both the laydown and construction areas. The new benchmarks were surveyed with a total station using permanent USGS (United States Geological Survey) benchmarks located at the NIST site for reference. All but one location was easily accessible for set-up and removal on an as needed basis. A semipermanent set-up with weatherproof housing was installed for the one transmitter that was difficult to access [see figure 4.]

A total of four transmitters were available for use during the field test. Although the receiving tool only uses two transmitters at a time during measurements, 


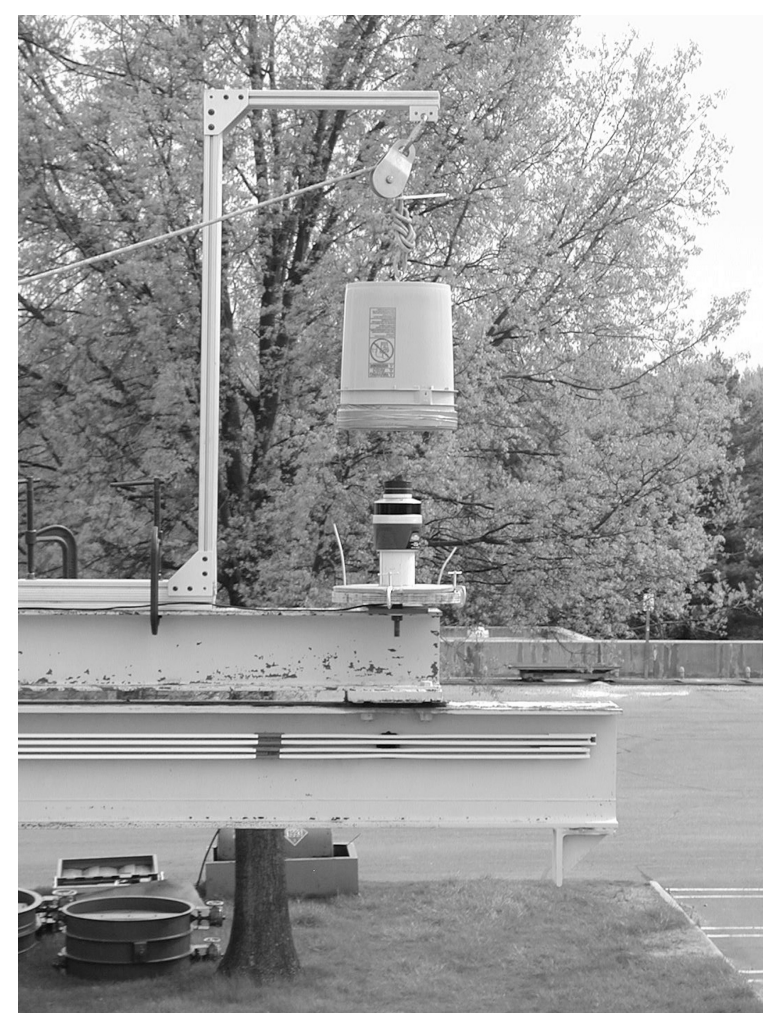

Figure 4. A pulley system and a long power cord allows the field inspector at ground level, approximately $12 \mathrm{~m}$ below, to lift the weatherproof cover and attach a rechargeable battery to begin using the transmitter for measurements.

having two "extras" significantly reduced the number of times transmitters needed to be moved. Prior to a measurement session, the field operator notes which benchmarks the beacons are set-up on to provide the site visualization system with the necessary coordinate frame information.

\subsection{Position and Orientation Determination}

The position and orientation of a part (referred to, collectively, as the part's "pose") is computed from the measured positions of 3 or more non-colinear fiducial points and the corresponding, nominal positions of those fiducial points in the part's coordinate frame (according to a pre-defined, geometric model of the part.)

The part-locator acts as both an information aggregator and a data-interpreter. It merges information from two different sources (sensor data from the field data collector) and part geometry (the fiducial-point location, from the geometric model.) From those two sets of data, the locator determines the part's position and orientation, which are derived quantities (and thus an interpretation of the "raw" data.) The part locator acts as separate service that can run on a different computer, communicating over a local-area network.
Each time the part locator service is used, it does three things:

1. Determines the part's pose (in some sitereferenced frame) from locations $(\mathrm{x}, \mathrm{y}, \mathrm{z})$ of fiducial points measured in that frame and corresponding data from the part's geometric model.

2. Computes a metric for the uncertainty in the pose.

3. Transforms the pose into the project's preferred coordinate frame (and representation) for storage in the project database.

In the locator, part's pose is primarily represented as a pose-parameter vector of 6 real numbers, $x, y, z$ (Cartesian translation) together with $\theta \mathrm{x}, \theta \mathrm{y}, \theta \mathrm{z}$ (rotations about site-fixed axes, with the rotations applied in $\theta \mathrm{x}, \theta \mathrm{y}, \theta \mathrm{z}$, order.)

$$
X=\left[x, y, z, \theta_{x}, \theta_{y}, \theta_{z}\right]^{T}
$$

The use of a 3-parameter representation for orientation has a potential problem common to 3parameter representations of 3-D orientation, namely numerical problems at (or near) the "gimbal lock" orientation (a singularity caused when the rotations result in allignment of two corresponding body-fixed axes, and the resultant loss of angular freedom about the remaining one.) This problem has not arisen in our data, so far. Use of a 4-parameter representation of orientation (e.g. quaternions) would require use of constrained optimization techniques to enforce the constraint required to keep the chosen 4-parameter representation geometrically valid.)

The parameter search technique for the "best-fit" pose parameters is based on unconstrained numerical optimization. In practice, the "best-fit" pose parameters do not represent a perfect fit of a pose vector to the measured data, due to measurement error, part variation (from the designed geometry), and finite-precision computation.

The cost function chosen for the minimizationbased search is a sum of squared errors between the measured and the corresponding (translated and rotated) model-derived points:

$$
J(X)=\sum_{i=1}^{n}\left|p_{\text {meas }}(i)-T(X) \cdot p_{\text {mod }}(i)\right|^{2}
$$

where

$\mathrm{J}(\mathrm{X})$ is the cost function,

$\mathrm{n}$ is the number of measured fiducial points, $\mathrm{T}(\mathrm{X})$ is a homogeneous transform that combines translation and rotation

$p_{\text {meas }}(i)$ is the $i^{\text {th }}(x, y, z)$ measurement

$p_{\text {mod }}(i)$ is the $i^{t h}(x, y, z)$ data from the model 
This cost is used for two reasons: First, a closedform solution for the gradient of the cost function is known; this can substantially speed up the optimization-based search. Second, this cost leads easily to a metric -- the root-mean-square error (between measured and "best-fit" of corresponding points) that has a physically-intuitive meaning. The RMS error indicates how far the measured fiducialpoint locations are (on average) from a perfect fit. As such, the RMS error is our current candidate for a metric of the uncertainty in the part's pose.

The best-fit pose parameter vector $\left(\mathrm{X}^{*}\right)$ is determined, using unconstrained numerical optimization of the cost function:

$$
X^{*}=\underset{X}{\operatorname{Min}}(J(X))
$$

The cost at the best fit, $\mathrm{J}^{*}=\mathrm{J}\left(\mathrm{X}^{*}\right)$, is then converted from the sum-of-squares-error to RMS (by normalizing by the number of points and taking the square root.) Finally, the pose is converted into the coordinate frame and representation required by the project's database and is then sent to the database.

\subsection{FUTURE WORK}

Upcoming work will begin to address some of the technological limitations experienced during field testing of the Comp-TRAK system on a live construction site. Research will focus on three specific areas:

1) Metrics for registration of multiple fanning laser transmitters: Development of a standard method for automatically registering large-scale laser-based coordinate measurement systems to the WGS-84 (World Geodetic System 1984) coordinate system. This work is essential to providing the basis for uniform and accurate coordinate registration.

2) Automated methods for fiducial point determination: Development of automated methods for extraction of easy-to-use fiducial points for structural steel elements. The current method of manually identifying fidicial points is a time consuming process, and difficulties arise when predefined points are inaccessible at the jobsite. Automated methods are needed to mine this information from existing data representations of structural steel parts.

3) Measures of performance for part locator algorithms: Data flow protocols being developed at NIST for transmission of sensor data from a construction site deliver a series of raw three dimensional coordinates for a structural component that has been placed on a construction site. There may be three (or more) 3-D points involved in an individual identification yet, by themselves, they do not describe the state of the object - i.e. its position and orientation (pose). Numerical algorithms have been developed at NIST to solve this problem using numerical optimization. Other approaches may be developed by industry in time. However, there is no standard method by which the accuracy of position and orientation (pose) determination can be measured or compared. Future work will focus on developing standard measures of performance for pose determination.

\section{Acknowledgements and Notices}

Special thanks to Jim Claytor, Chuck Helm, and Mike Dalton of Green Contracting Company, and K.C. Patel and Carrie Kelly of the NIST Plant Division.

Certain company products are mentioned in the text in order to adequately specify the equipment used. In no case does such an identification imply recommendation or endorsement by the National Institute of Standards and Technology, nor does it imply that the products are necessarily the best available for the purpose.

\section{REFERENCES}

[1] Furlani, K.M, and Stone, W.C., "Architecture for Discrete Construction Component Tracking," Proceedings, 16th IAARC/IFAC/IEEE International Symposium on Automation and Robotics in Construction, September 22-24, 1999 , Madrid, Spain, pp 289-294.

[2] Stone, W.C., Furlani, K., and Pfeffer, L., "Automated Part Tracking on the Construction Site," Proceedings, $4^{\text {th }}$ International Conference on

Robotics for Challenging Situations and Environments (Robotics 2000), Albuquerque, New Mexico, February 27-March2, 2000.

[3] VIA, Inc., 12550 W. Frontage Rd. \#201, Burnsville, MN, 55337, http://www.flexipc.com.

[4] Arc-Second (1999), ArcSecond, Inc., 44880 Falcon Pl. \#100, Dulles, VA 20166, http://www.arcsecond.com.

[5] Pfeffer, L.E., and Latimer, D.T., (1999), “Toward Open Network Data-Exchange Protocols for Construction Metrology and Automation: LIVEVIEW," Proceedings, 16th IAARC/IFAC/IEEE International Symposium on Automation and Robotics in Construction, September 22-24, 1999 , Madrid, Spain, pp 117-122.

[6] Furlani, K., Pfeffer, L., Latimer, D., and Stone, W.C., (1999), NIST Construction Automation Program Report No. 5: Real-Time Vehicle and Discrete Component Tracking for Construction, National Institute of Standards and Technology, Gaithersburg, MD 20899, USA. 